\title{
DESIGN AND ANALYSIS OF MICROSTRIP SISO, SIMO AND MIMO ANTENNA
}

\author{
MANOJ PITALE ${ }^{1} \&$ NITESH KUMAR ${ }^{2}$ \\ ${ }^{1}$ Department of Electronics and Communication, R.G.P.V. University, Bhopal Madhya Pradesh, India \\ ${ }^{2}$ Department of Electronics and Communication, S.I.R.T.S. College, Bhopal Madhya Pradesh, India
}

\begin{abstract}
In this new era a lot of work done in the field microstrip MIMO antenna, but mutual coupling is the significant area which requires improvement. In this paper, we tend to reduce the problem of mutual coupling with the assistance of the proposed decoupling element. We also design and analyzed the proposed antenna design on a network analyzer and compare it with the simulated result. Along with MIMO antenna, we also design and analyze SISO and SIMO antennas. The center frequencies of these antennas are at $2.3 \mathrm{GHz}$.
\end{abstract}

KEYWORDS: Microstrip MIMO Antenna, SISO, SIMO, MIMO, Decoupling Element, Resonator \& Microstrip Patch Antenna

Received: Feb 26, 2018; Accepted: Mar 15, 2018; Published: Mar 31, 2018; Paper Id.: IJECIERDAPR20182

\section{INTRODUCTION}

In today's era communication become a crucial factor in life and thence communication system is additionally needed to enhance. There is a lot of work is being projected for enhancing the communication system like increasing of bandwidth, gain, channel capacity, reliability, etc. In the previous few years, researchers are showing significant interest towards MIMO (Multiple-Input Multiple-Output). MIMO helps to improve the reliability, channel capacity, multipath propagation, enhanced data throughput, higher data rates and the most vital is its wide application in Wi-Fi LAN, Bluetooth, UMTS and in next-generation communication (5G) [I].

In radio, MIMO could be a methodology for multiplying the capability of a communication system using multiple transmit and receive antenna to take advantage of multipath propagation. In wireless multipath could be a development that leads to radio signals reaching the receiving antenna by 2 or more than 2 ways. MIMO especially refers to a sensible technique for sending and receiving more than one information signal at the same time over an equivalent radio channel with the assistance of multipath propagation. The main aim of developing MIMO is to boost the performance of the single information signal [II].

As there are so many advantages of MIMO antenna there are also having some limitations like these types of antennas are quite complex, having high power consumptions as compared to the other microstrip patch antenna, these antennas are quite costly than the microstrip patch antenna and the main limitation is "Mutual Coupling" between antenna elements. Between antenna components, mutual coupling is described as the energy absorbed by one antenna receiver once another near antenna component is operating, that cause signal interference between them and so the higher the value of mutual coupling degrades the antenna potency. The more energy absorbed by the near antenna component, the less are going to be transmitted for communication to the far field antenna with which we wish to communicate[III]. For reducing the mutual coupling between multiple channels of microstrip MIMO antenna, various numbers of works were presented in the literature. In literature, the main methods which are used for reducing the mutual coupling have Defected Ground 
Structure (DGS), Electronic Band Gap (EBG) Structure and Resonators.

In this paper, we have simulated the three cases of MIMO antenna, (SISO, SIMO \& MIMO) and analyze them on Network Analyzer to compare the actual results with the simulated results. Where SISO, SIMO \& MIMO stands for Single Input Single Output, Single Input Multiple Output, Multiple Input Multiple Output respectively. The problem of mutual coupling in microstrip MIMO antenna is also resolved by using decoupling element. We have used "I Slotted resonator" as a decoupling element. In this paper, we also simulated and analyze the different positions, one is when are channels are placed Vertical to each other and one is when channels are placed horizontal to each other, of the microstrip MIMO antenna along with this we also test the two different combinations, one is of two channels two ports and another one is about four channels two ports, of the microstrip MIMO antenna. All the simulation work is done in CST 2016 software.

In the $2^{\text {nd }}$ fragment, the design of antennas and results has been shown. The $3^{\text {rd }}$ part is the summary of this paper along with the comparison of simulated and analyzed results.

\section{ANTENNA DESIGNS AND RESULTS}

As discussed in the first section in this paper, we designed and analyzed the three cases of MIMO antenna i.e. SISO SIMO \& MIMO. The patch antenna is prepared by the set of formulas [IV] used for calculating of width and length of microstrip patch antenna which is as follows:

The width of the patch $(w)$ is given by

$$
w=\frac{C}{2 f_{0} \sqrt{\frac{\epsilon_{r}+1}{2}}}
$$

Here $\mathrm{C}$ is the velocity of light which is $3 \times 10^{8} \mathrm{~m} / \mathrm{s}, f_{0}$ is the frequency and $\epsilon_{r}$ is the dielectric constant. In this paper, we use FR-4 substrate and hence the value of $\epsilon_{r}$ is 4.4 .

Effective width

$$
W_{\text {eff }}=w+2 \Delta W
$$

Here $\Delta W=1.631 \ln \left(\frac{4}{\pi}\right)$

Length of the patch $(L)$ is:

$$
L=L_{e f f}-2 \Delta L
$$

The effective length $L_{e f f}=\frac{C}{2 f_{0} \sqrt{\epsilon_{\text {reff }}}}$

Here $\epsilon_{\text {reff }}$ is the effective dielectric constant which is given by

$$
\epsilon_{\text {reff }}=\frac{\epsilon_{r}+1}{2}+\frac{\epsilon_{r}-1}{2}\left[1+\frac{12 h}{w}\right]^{-\frac{1}{2}}
$$

Here $w$ is the width of the patch and $h$ is the height.

Normalized extension of the length $\Delta L$ is 


$$
\frac{\Delta L}{h}=0.412\left\{\frac{\left(\epsilon_{\text {reff }}+3\right)\left(\frac{w}{h}+0.264\right)}{\left(\epsilon_{\text {reff }}-0.258\right)\left(\frac{w}{h}+0.8\right)}\right\}
$$

For the dominant $T M_{010}$ mode, the resonant frequency of the microstrip patch antenna is a function of its length [IV] and it is given by

$$
\left(f_{r}\right)_{010}=\frac{1}{2 L \sqrt{\epsilon_{r}} \sqrt{\mu_{0} \epsilon_{0}}}=\frac{v_{0}}{2 L \sqrt{\epsilon_{r}}}
$$

Where $v_{0}$ is the speed of light in the free space and is given by :

$$
v_{0}=\frac{1}{\sqrt{\mu_{0} \epsilon_{0}}}
$$

Since the $\left(f_{r}\right)_{010}$ does not account for fringing, it must be modified to include edge effects and should be computed using:

$$
\left(f_{r c}\right)_{010}=\frac{1}{2 L_{e f f} \sqrt{\epsilon_{r}} \sqrt{\mu_{0} \epsilon_{0}}}=\frac{1}{2(L+2 \Delta L) \sqrt{\epsilon_{r}} \sqrt{\mu_{0} \epsilon_{0}}}=q \frac{v_{0}}{2 L \sqrt{\epsilon_{r}}}
$$

Where

$$
q=\frac{\left(f_{r c}\right)_{010}}{\left(f_{r}\right)_{010}}
$$

The $\mathrm{q}$ factor is referred to as the fringe factor.

For the efficient radiator, a practical width that leads to better radiation efficiency is [IV]

$$
w=\frac{1}{2 f_{r} \sqrt{\mu_{0} \epsilon_{0}}} \sqrt{\frac{2}{\epsilon_{r}+1}}=\frac{v_{0}}{2 f_{r}} \sqrt{\frac{2}{\epsilon_{r}+1}}
$$

In the same manner, the actual practical length is determined by [IV]

$$
L=\frac{1}{2 f_{r} \sqrt{\mu_{0} \epsilon_{0}}}-2 \Delta L
$$

After the successful construction of patch, the next procedure is to provide feed to the antenna. Feeding is mainly used as for conversation purpose in the antenna, the conversation of the radio signal into an electrical signal or an electric signal into a radio signal and hence it is considered to be a part of the antenna [V]. There are primarily four kinds of feedings are utilized in microstrip patch antenna Microstrip Line Feed, Coaxial Probe Feed, Aperture Coupled Feed, and Proximity Coupled Feed. Among these feedings, we are using Microstrip Line Feeding for our proposed antennas. It is the simplest form of feeding and very easy to fabricate.

\subsection{SISO ANTENNA}

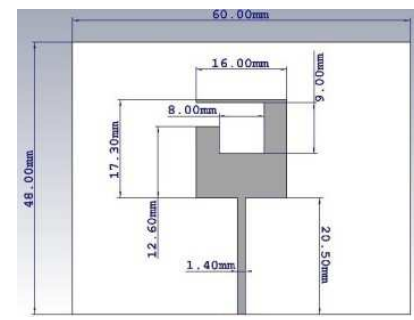

Figure 2(a): Antenna 1: Design Dimensions of Proposed SISO Antenna 


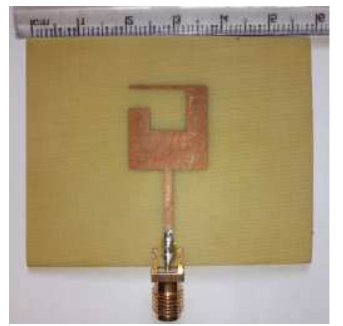

Figure 2(b): Antenna 1: Photograph of Hardware

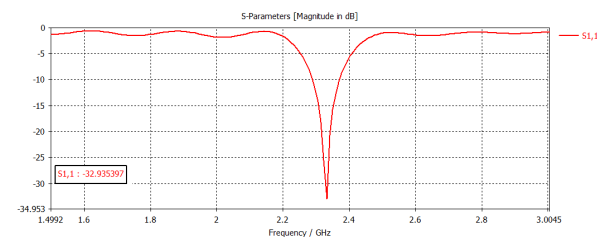

Figure 2(c): Antenna 1: Simulated Result (Graph of S- Parameter)

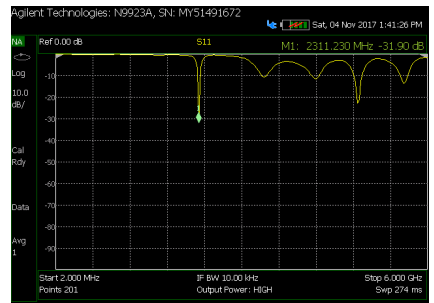

Figure 2(d): Antenna 1: Analyzed Result

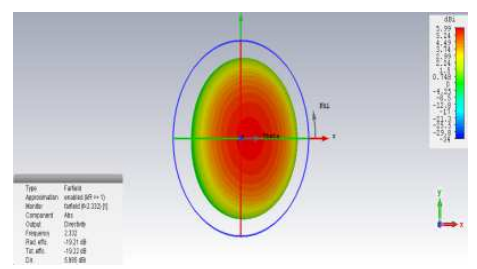

Figure 2(e): Antenna 1: Farfield

The SISO (SINGLE INPUT SINGLE OUTPUT) antenna is the simplest form of MIMO antenna. It is a low profile microstrip patch antenna with less complexity and simple to manufacture and cost-efficient [IV]. The proposed antenna design, dimensions, hardware photograph along with a graph of S-parameter and far-field are shown in the figure. 2. The proposed antenna works at $2.3 \mathrm{GHz}$ frequency and has a return loss around $-33 \mathrm{~dB}(-32.94 \mathrm{~dB})$.

The SISO antenna is most commonly used in radio, GSM and CDMA systems. But as the technology upgraded the recruitment of the higher data rate, better Bit Error Rates are required which does not fulfill by the SISO system.

\subsection{SIMO ANTENNA}

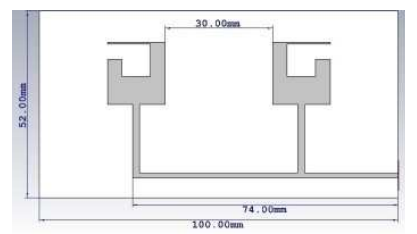

Figure.3 (a): Antenna 2: Design Dimensions of Proposed SIMO Antenna 


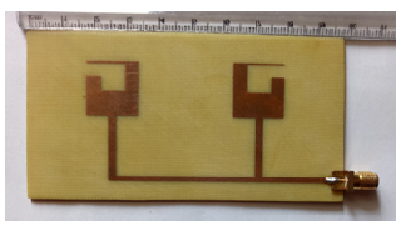

Figure.3 (b): Antenna 2: Photograph of Hardware

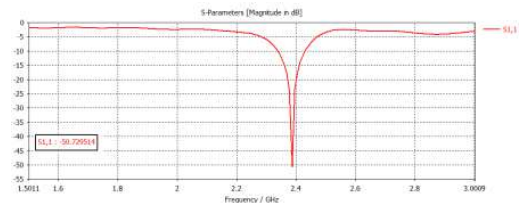

Figure.3 (c): Antenna 2: Result (Graph of S- Parameter)

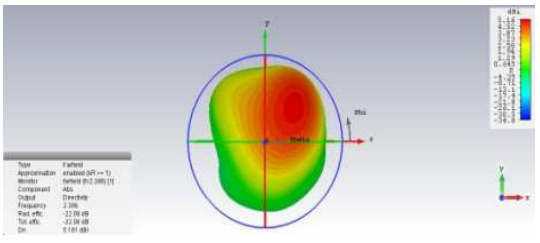

Figure.3 (d): Antenna 2: Farfield

The Single Input Multiple Outputs antenna is an advanced form of SISO antenna. This antenna is slightly more complex as compare to SISO antenna because it has multiple channels. The proposed SIMO antenna, its S-parameter graph, and far-field are shown below (figure. 3). This antenna has much better gain as compared to SISO antenna. It has a return loss around $-51 \mathrm{~dB}(-50.73 \mathrm{~dB})$ and works at $2.39 \mathrm{GHz}$ frequency.

As the SIMO antenna design is complex then SISO is one of the disadvantages, but it is able to improve error performance over fading multipath channels by providing spatial diversity [VI]. It is used in a WCDMA system because it provides higher data rate than the SISO system. It is also applicable to Digital television, MANs but still it is unable to provide good receive reception and hence it is not used in the next generation communication system.

\subsection{MIMO ANTENNA}

The Multiple Inputs Multiple Outputs antenna has more than one transmitting and receiving channels. This antenna works on multipath propagation phenomena due to which the probability of receiving reception increased. It also increases the data throughput and has very low Bit Error Rate as compared to the SISO and SIMO antenna. MIMO Antenna uses the spatial multiplexing technique for increasing the channel capacity. In spatial multiplexing higher rate signal is split into many lower rate streams and, every stream is transmitted from the various transmitting antennas on the same frequency channel [VII].

By exploiting spatial multiplexing MIMO antenna is able to realize higher data rates over the long distance with the assistance of MIMO-OFDM. This method support increased data output even underneath condition of interference, signal attenuation and multipath. This is the reason why it is most usually used in the next generation communication system.

As MIMO antenna is having the upper hand as compare to the other microstrip patch antennas it has some disadvantages also like as there are multiple channels the design of MIMO antenna becomes more complex as well as it also increases the overall cost of the antenna. One major problem with the MIMO antenna is Mutual Coupling. As the 
MIMO antenna has multiple channels which are placed closely to each other and having multiple inputs as well it is the main reason for mutual coupling between antenna channels. This mutual coupling is the main reason for the reduction in gain and distorted beam pattern.

In the past few years, various works were proposed to reduce this Mutual Coupling by using DGS, EBG structure or by the use of resonators. DGS and EBG structures placed within the ground plane offer undesirable back radiation. By the employment of these techniques, mutual coupling has been reduced significantly at the cost of any sophisticated structure of the ground plane and it suffers from radiation pattern degradation [VIII]. The DGS and EBG structures are more complicated structures and optimum designs are more complicated to realize [IX] and hence in this paper, we use "I slotted resonator for reducing the mutual coupling" as a decoupling element. This decoupling element is designed in such a way so that the compactness of the MIMO system can be kept as much as possible.

\subsubsection{DECOUPLING ELEMENT}

The basic concept for reducing the Mutual Coupling is the presence of one or more decoupling element or networks. The decoupling components are situated between neighboring channels of MIMO antenna. These decoupling components alleviate the mutual coupling problem between closely spaced antenna elements $[\mathrm{X}]$. This decoupling element resists the signal going straightforwardly from component to component. Here we should note that the inserted decoupling element does not degrade the antenna's radiation pattern, return loss, gain. In this paper, we use an I slotted resonator as a decoupling element which is used for reducing the mutual coupling between the channels of microstrip MIMO patch antenna.

\subsubsection{MIMO DESIGN}

In this paper, we proposed two types of MIMO antenna one is two channels two ports MIMO antenna and another one is four channel two ports MIMO antenna. There are more two proposed antennas in two channels two ports MIMO antenna one is when proposed two G shaped channels are placed to each other horizontally and another one is when proposed two G shaped channels are placed to each other vertically. The four-channel two ports MIMO antenna is formed from the proposed SIMO antenna design.

In the two channels two ports MIMO antenna when both the channels are placed each other to horizontal as shown in the figure. 4 (a) both the channels of MIMO antenna are placed to each other very closely, due to closely spacing between antenna channels mutual coupling occurs and hence only 1 channel is operating ( Figure. 4(c)) from far-field figure (figure. $4(\mathrm{~d})$ ) the directivity of the antenna is $5.883 \mathrm{dBi}$ and from simulation VSWR is 1.13 .

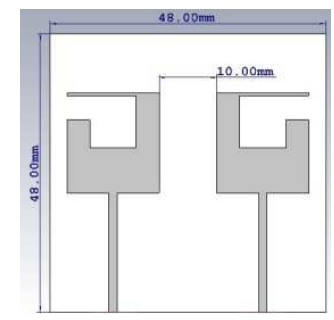

Figure.4 (a): Antenna 3: Design Dimensions of Proposed MIMO Antenna (without Resonator) 


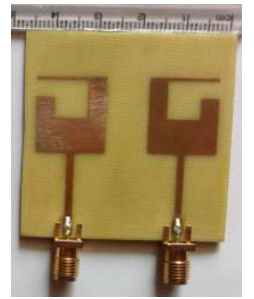

Figure.4 (b): Antenna 3: Photograph of Hardware

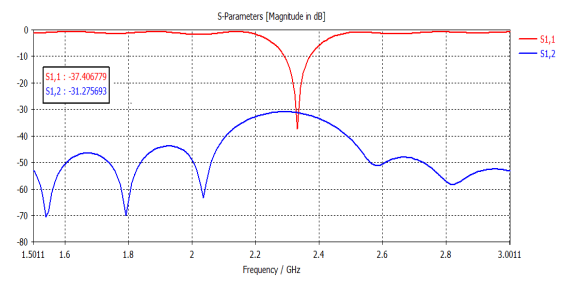

Figure.4 (c): Antenna 3: Simulated Result (Graph of S- Parameter)

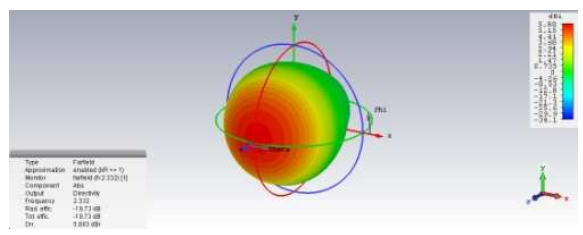

Figure.4 (d): Antenna 3: Fairfield

To reduce the mutual coupling between the channels of MIMO antenna in Figure. 4 (a) a decoupling element is placed between the channels of MIMO antenna channels (Figure. 5 (a)) due to this placement of decoupling element i.e. I slotted resonator mutual coupling reduces by $34.72 \mathrm{~dB}$ and from the far-field figure (figure. 5(d)) the directivity of the antenna is $5.735 \mathrm{dBi}$. And the simulated result of VSWR is 1.03 .

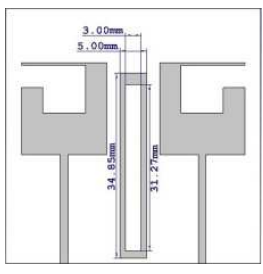

Figure.5 (a): Antenna 4: Design Dimensions of Proposed MIMO Antenna (with Resonator)

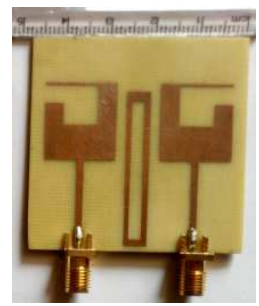

Figure.5 (b) Antenna 4: Photograph of Hardware 


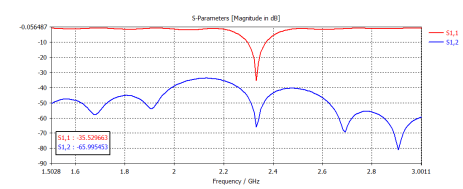

Figure.5 (c) Antenna 4: Simulated Result (Graph of S- Parameter)

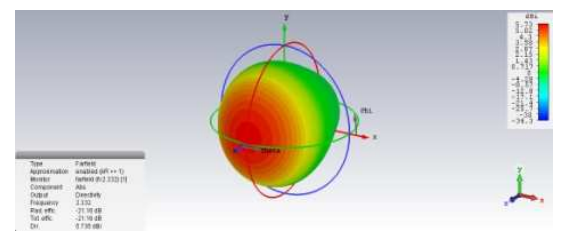

Figure.5 (d): Antenna 4: Farfield

Similarly, in two ports two channels MIMO antenna when channels are placed vertically to each other only one channel is operating because of mutual coupling and the VSWR of the antenna is 1.02 and from the far-field diagram, the directivity of the antenna is $4.89 \mathrm{dBi}$ (figure. 6). To reduce the mutual coupling a decoupling element I slotted resonator is used and due to this the $26.92 \mathrm{~dB}$ reductions calculated between MIMO antenna 5 and antenna 6 . The VSWR of the antenna 6 is 1.05 and directivity is $4.872 \mathrm{dBi}$ (figure. 7).

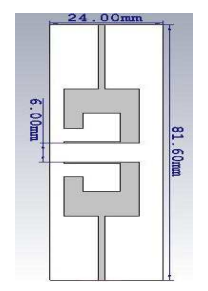

Figure.6 (a): Antenna 5: Design Dimensions of Proposed MIMO Antenna (without Resonator)

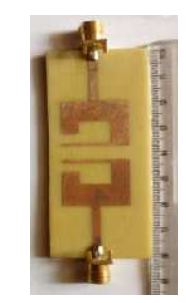

Figure.6 (b): Antenna 5: Photograph of Hardware

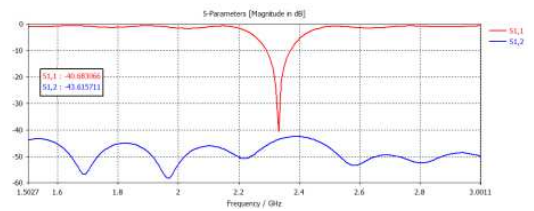

Figure.6 (c): Antenna 5: Simulated Result (Graph of S- Parameter)

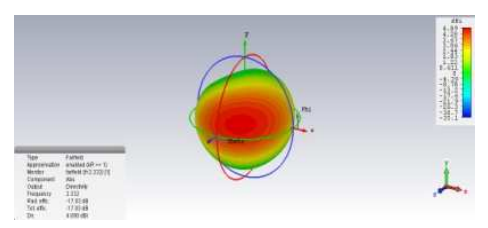

Figure.6 (d): Antenna 5: Farfield 


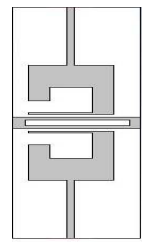

Figure.7 (a): Antenna 6: Design Dimensions of Proposed MIMO Antenna (with Resonator)

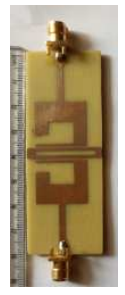

Figure.7 (b): Antenna 6: Photograph of Hardware

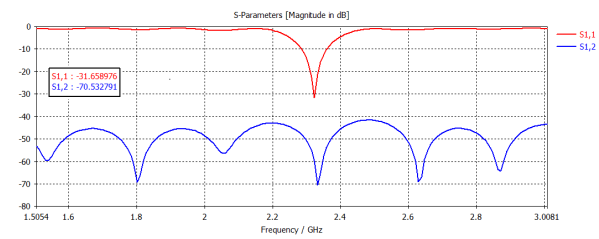

Figure.7 (c): Antenna 6: Simulated Result (Graph of S- Parameter)

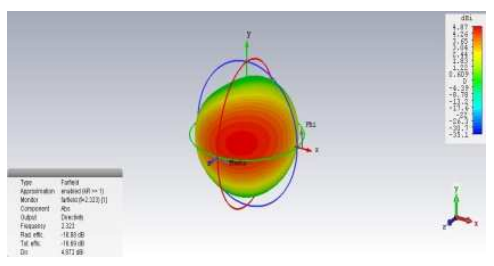

Figure.7 (d): Antenna 6: Far-field

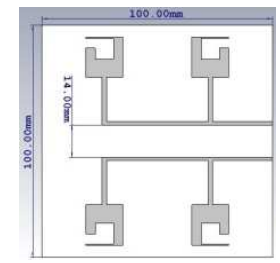

Figure.8 (a): Antenna 7: Design Dimensions of Proposed MIMO Antenna (without Resonator)

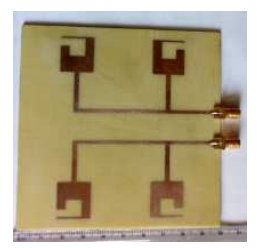

Figure.8 (b): Antenna 7: Photograph of Hardware 


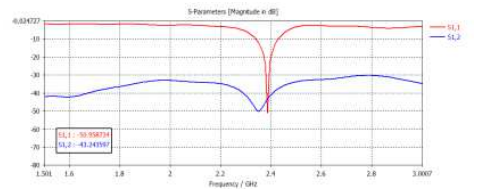

Figure.8 (c): Antenna 7: Simulated Result (Graph of S- Parameter)

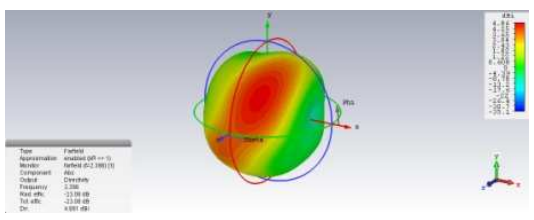

Figure.8 (d): Antenna 7: Far-field

In figure. 8, antenna 7 is a MIMO antenna in the form of two SIMO antennas placed closely to each other and to reduce mutual coupling in antenna 7, I slotted resonator (decoupling element) is placed as shown in the figure. 9 (antenna 8), due to this $23.63 \mathrm{~dB}$ reduction is calculated in a mutual coupling. The VSWR and directivity of antenna 7 is 1.01 and $4.861 \mathrm{dBi}$ respectively. And for antenna 8 VSWR is 1.02 and directivity is $5.028 \mathrm{dBi}$.

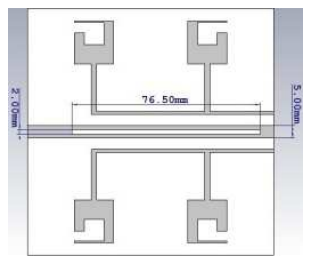

Figure.9 (a): Antenna 8: Design Dimensions of Proposed MIMO Antenna (with Resonator)

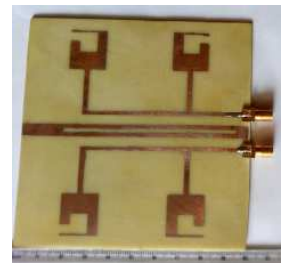

Figure.9 (b): Antenna 8: Photograph of Hardware

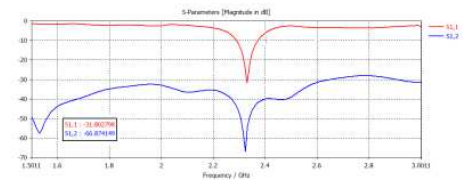

Figure.9 (c): Antenna 8: Simulated Result (Graph of S- Parameter)

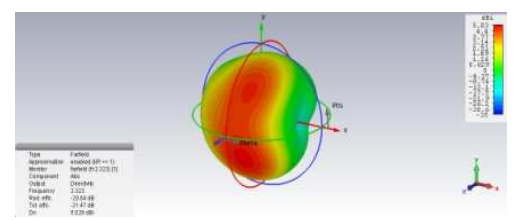

Figure.9 (c): Antenna 8: Far-field 


\section{CONCLUSIONS}

The proposed SISO antenna has simulated return loss of $-32.94 \mathrm{~dB}$ and the analyzed results of SISO antenna is $31.9 \mathrm{~dB}$. In the proposed SIMO antenna the simulated result of return loss shows the value of $-50.73 \mathrm{~dB}$, but at the analyzer, the proposed SIMO antenna gives the value of $-48.8 \mathrm{~dB}$. The proposed MIMO antenna without and with decoupling element was also simulated and tested on a network analyzer. As mentioned in the previous section the problem of mutual coupling and its reduction with the help of decoupling element is proposed which reduces the mutual coupling at a great level. In this section, we compare the simulated result with analyzed results of all proposed antennas in the table1.

Table 1: Simulated v/s Analyzed Result with Error \% of Return Loss

\begin{tabular}{|c|c|c|c|c|c|c|}
\hline \multirow[b]{2}{*}{$\begin{array}{l}\text { S. } \\
\text { No. }\end{array}$} & \multirow{2}{*}{$\begin{array}{c}\text { Design } \\
\text { Description }\end{array}$} & \multicolumn{2}{|c|}{ Simulated Result } & \multicolumn{2}{|c|}{ Analyzed Result } & \multirow{2}{*}{$\begin{array}{c}\text { Error \% } \\
\text { (of Return Loss) }\end{array}$} \\
\hline & & $\begin{array}{c}\text { Center } \\
\text { freq. }(\mathrm{GHz})\end{array}$ & $\begin{array}{c}\text { Return Loss } \\
\text { (dB) }\end{array}$ & $\begin{array}{c}\text { Center Freq. } \\
(\mathrm{GHz})\end{array}$ & $\begin{array}{c}\text { Return Loss } \\
\text { (dB) }\end{array}$ & \\
\hline 1. & $\begin{array}{c}\text { Antenna } 1 \\
\text { (SISO) }\end{array}$ & 2.3 & -32.94 & 2.3 & -31.90 & 3.16 \\
\hline 2. & $\begin{array}{c}\text { Antenna } 2 \\
\text { (SIMO) }\end{array}$ & 2.3 & -50.73 & 2.4 & -48.8 & 3.80 \\
\hline \multirow{2}{*}{3.} & \multirow{2}{*}{$\begin{array}{c}\text { Antenna } 3 \\
\text { (MIMO) }\end{array}$} & \multirow{2}{*}{2.3} & $S_{1,1}=-37.41$ & \multirow{2}{*}{2.3} & $S_{1,1}=-36.13$ & \multirow{2}{*}{3.42} \\
\hline & & & $S_{1,2}=-31.28$ & & $S_{1,2}=-29.09$ & \\
\hline \multirow{2}{*}{4.} & \multirow{2}{*}{$\begin{array}{c}\text { Antenna } 4 \\
\text { (MIMO) }\end{array}$} & \multirow{2}{*}{2.3} & $S_{1,1}=-35.53$ & \multirow{2}{*}{2.5} & $S_{1,1}=-35.05$ & \multirow{2}{*}{1.35} \\
\hline & & & $S_{1,2}=-66$ & & $S_{1,2}=-65.43$ & \\
\hline \multirow{2}{*}{5.} & \multirow{2}{*}{$\begin{array}{l}\text { Antenna } 5 \\
\text { (MIMO) }\end{array}$} & \multirow{2}{*}{2.3} & $S_{1,1}=-41.83$ & \multirow{2}{*}{2.2} & $S_{1,1}=-39.12$ & \multirow{2}{*}{6.48} \\
\hline & & & $S_{1,2}=-43.62$ & & $S_{1,2}=-40.6$ & \\
\hline \multirow{2}{*}{6.} & \multirow{2}{*}{$\begin{array}{l}\text { Antenna } 6 \\
\text { (MIMO) }\end{array}$} & \multirow{2}{*}{2.3} & $S_{1,1}=-31.66$ & \multirow{2}{*}{2.2} & $S_{1,1}=-28.65$ & \multirow{2}{*}{9.51} \\
\hline & & & $S_{1,2}=-70.53$ & & $S_{1,2}=-66.43$ & \\
\hline \multirow{2}{*}{7.} & \multirow{2}{*}{$\begin{array}{c}\text { Antenna } 7 \\
\text { (MIMO) }\end{array}$} & \multirow{2}{*}{2.3} & $S_{1,1}=-50.96$ & \multirow{2}{*}{2.3} & $S_{1,1}=-49.95$ & \multirow{2}{*}{1.98} \\
\hline & & & $S_{1,2}=-43.24$ & & $S_{1,2}=-40.06$ & \\
\hline \multirow[b]{2}{*}{8.} & \multirow{2}{*}{$\begin{array}{l}\text { Antenna } \\
\text { (MIMO) }\end{array}$} & \multirow[b]{2}{*}{2.3} & $S_{1,1}=-31.80$ & \multirow[b]{2}{*}{2.3} & $S_{1,1}=-31.19$ & \multirow[b]{2}{*}{1.92} \\
\hline & & & $S_{1,2}=-66.87$ & & $S_{1,2}=-65.86$ & \\
\hline
\end{tabular}

From the table, it is seen that in the antenna 4, $6 \& 8$ the mutual coupling is reduced in the simulated result by $34.72 \mathrm{~dB}, 26.92 \mathrm{~dB} \& 23.63 \mathrm{~dB}$ respectively, while in analyzed result the mutual coupling is reduced by $34.34 \mathrm{~dB}, 25.83 \mathrm{~dB}$ and $22.8 \mathrm{~dB}$ respectively. After the comparison of simulated and analyzed result, we found the best result which is comparably higher than the work presented in [XI]. In future, we will try to improve the return loss of four channels two ports MIMO antenna. Table 2 shows the VSWR and Directivity of the all antennas.

Table 2: VSWR and Directivity of All Antennas

\begin{tabular}{|c|c|c|c|c|c|c|c|}
\hline Sr. No. & Antenna & VSWR & Directivity (in dBi) & Sr. No. & Antenna & VSWR & Directivity (in dBi) \\
\hline 1 & Antenna 1 & 1.11 & 5.985 & 5 & Antenna 5 & 1.02 & 4.890 \\
\hline 2 & Antenna 2 & 1.01 & 5.161 & 6 & Antenna 6 & 1.05 & 4.872 \\
\hline 3 & Antenna 3 & 1.13 & 5.883 & 7 & Antenna 7 & 1.01 & 4.861 \\
\hline 4 & Antenna 4 & 1.03 & 5.735 & 8 & Antenna 8 & 1.02 & 5.028 \\
\hline
\end{tabular}




\section{ACKNOWLEDGEMENT}

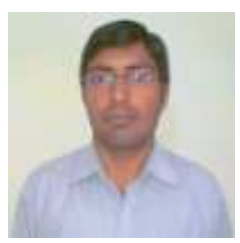

The analyses of proposed antenna designs are analyzed on Agilent Technologies N9923A network analyzer in the Maulana Azad National Institute of Technology (M.A.N.I.T.), Bhopal (M.P.), India under the guidance of Dr. O. P. Meena, associate professor, Electronics and Communication department, M.A.N.I.T., Bhopal (M.P.).

\section{REFERENCES}

1. Ogale, A., Chaoudhary, S., \& Patil, A. J. (2013). Performance Evaluation of MIMO-OFDM System using Matlab® Simulink With Real Time Image Input. Tenth International Conference on Wireless and Optical Communications Networks, 1-5.

2. Thrimurthulu, V., \& Murti Sarma, N. S. (2017). Fading Mitigation Techniques in Wireless Mobile Communication Systems. International Journal of Engineering Technology Science and Research, 4.

3. Shiu, D. S., Foschini, G. J., \& Kahn, M. J. (2000). Fading correlation and its effect on the capacity of multi-element antenna systems. IEEE Trans Commun,48,502-513.

4. Ashwini C Tengli \& P. M. Hadalgi, Design of Inset-Fed Triple-Band Rectangular Microstrip Antenna for Wimax, 5.5 Ghz Wlan and Lower X-Band Satellite Applications, International Journal of Electrical and Electronics Engineering Research (IJEEER), Volume 7, Issue 4, July - August 2017, pp. 29-36

5. Constantine A. Balanis. (1982). Antenna Theory: Analysis and Design. New Jersey: John Wiley and Sons, Inc.publication.

6. Mandal, A., Ghosal, A., \& et.al.(2012). Analysis of Feeding Techniques of Rectangular Microstrip Antenna. IEEE International Conference on Signal Processing, Communication and Computing, 26-31.

7. Nagraj, S., \& Xiao, J.(2010). Best antenna selection for coded SIMO-OFDM. Elsvier Signal Processing, 90, 391-394.

8. Singh, Y.P., \& Sharma, R. (2015). Review on Spatial Multiplexing in Modern MIMO Systems: An Innovative Technology. IJECT, 6.

9. Ghosh, K., \& Parui, S. K. (2013). Reduction of mutual coupling between e-shaped microstrip antennas by using a simple microstrip I-section. Microwave and Optical Technology Letters, 55.

10. Farsi, S., Aliakbarian, H., \& et. al. (2012). Mutual coupling reduction between planner antennas by using a simple U- section. IEEE antennas and wireless propagation letters, 11 .

11. Coetzee, J.C., \& Yu, Y. (2008). closed form design equation for decoupling network of small array. Electronics Letters, 44.

12. Ghafor, A., Hameed, A. A., \& et.al. (2015). Mutual Coupling Reduction of a (2×1) MIMO Antenna System Using Parasitic Element Structure for WLAN Applications. Journal of Emerging Trends in Computing and Information Sciences, 


\section{AUTHOR DETAILS}

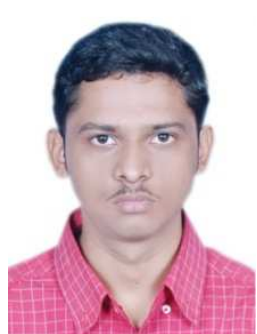

Manoj Pitale has done his B.E. In Stream Electronics and communication engineering from RGTU Bhopal in 2014. Currently, he is pursuing M.Tech. in Digital Communication from RGTU, Bhopal, India. His research interest includes Microstrip patch antenna, resonators and image processing.

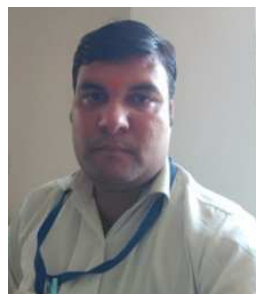

Nitesh Kumar has done his B.Tech. In Stream Electronics and communication engineering from UPTU in 2006. He has received his M.E. Degree in Microwave from J.E.C., Jabalpur, India in 2012. Presently he is the assistant professor at Sagar Institute of Research, Technology \& Science in Electronics and communication department since July 2012, His research interest includes microwave filters, Microstrip patch, antenna and resonators. 
